\title{
FIXED POINT THEOREMS FOR CERTAIN CLASSES OF SEMIGROUPS OF MAPPINGS
}

\author{
BY \\ MO TAK KIANG(1)
}

\begin{abstract}
Fixed point theorems for commuting semigroups of self-mappings are considered in this paper. A generalization of the classical Markov-Kakutani theorem is first given. This is followed by a fixed point theorem for commutative semigroups of continuous asymptotically-nonexpansive self-mappings on a weakly compact, convex subset of a strictly convex Banach space.
\end{abstract}

The Markov-Kakutani theorem [8], [7] asserts the existence of a common fixed point of a semigroup $\exists$ of continuous affine self-mappings on a compact convex subset of a Hausdorff linear topological space. It is shown that the conclusion of this theorem holds with a weaker assumption. While no element of $\exists$ need be affine, it is sufficient to guarantee the existence of a common fixed point when $\rightarrow$ is continuous and asymptotically-affine. This result also gives some additional information on the location of the common fixed point.

In the case when the space in question is Banach, De Marr [2] has obtained a similar result for nonexpansive mappings. This result is further generalized by Holmes [4] (cf. also [5]) to the case when 7 is continuous and asymptoticallynonexpansive. All these results concern self-mappings on a compact convex subset of a Banach space. A fixed point theorem for a commutative semigroup of continuous asymptotically-nonexpansive self-mappings on a weakly compact, convex subset of a strictly convex Banach space is also obtained. For weakly compact convex subsets with normal structure, this result also provides some additional information on its location. Under these conditions, the centre (in the sense of Brodskii and Milman [1]) of the closed convex hull of the orbit of a certain point is such a fixed point. A similar result is obtained in the case when the convex subset in question is compact (cf. Holmes' result mentioned above which merely asserts the existence of a common fixed point). A counterexample is included to show that when strict convexity is deleted, the conclusion of this result concerning the location of the existing fixed point no longer holds.

Received by the editors November 21, 1971.

AMS (MOS) subject classifications (1970). Primary 47H10.

Key words and phrases. Common fixed points, asymptotically-affine, asymptotically-nonexpansive, normal structure.

(1) This work was supported by a National Research Council of Canada Scholarship and NRC Grant A-8756.

The contents of this paper form part of the author's doctoral dissertation at Dalhousie University. The dissertation was prepared under the direction of Professor M. Edelstein whom the author wishes to thank for his encouragement and advice. 
We conclude this paper with a method suggested by the above counterexample to locate common fixed points for commuting semigroups of continuous asymptotically-nonexpansive self-mappings when the Banach space in question is not necessarily strictly convex.

1. A mapping $f$ of a linear space $X$ into itself is called affine if $f[\lambda x+(1-\lambda) y]$ $=\lambda f(x)+(1-\lambda) f(y)$ for every $x, y \in X$ and all $\lambda \in[0,1]$.

A family of self-mappings $\exists$ on a linear space $X$ is called affine if each $f \in \mathcal{Y}$ is affine. $\rightarrow$ is called asymptotically-affine if for every $x, y \in X$, there exists $g \in \mathcal{G}$ such that

$$
f g[\lambda x+(1-\lambda) y]=\lambda f g(x)+(1-\lambda) f g(y) \text { for every } f \in \mathcal{G}
$$

and all $\lambda \in[0,1]$. In such a case, we call $x, y$ asymptotically-affine with respect to g.

It is clear that the notion that $\rightarrow$ be asymptotically-affine is a generalization of the notion that $\Im$ be affine. (Examples of commuting semigroups which are asymptotically-affine but not affine are given in \$4.)

Let $X$ be a metric space with metric $d$ and $\Im$ a family of self-mappings on $X$. Let $z \in X$. The set $\mathcal{Y}(z)=\{z\} \cup\{f(z): f \in \mathcal{Y}\}$ is called the orbit of $z$.

1.1. Theorem 1 (Markov-Kakutani [8], [7]). Let $X$ be a compact convex subset of a Hausdorff linear topological space, and $\exists$ be a commutative semigroup of continuous affine self-mappings on $X$. Then $\exists$ has a common fixed point.

Before proving Theorem 2, which is a generalization of Theorem 1, we prove first the following simple lemmas on which the proof of the main theorem is based.

Lemma 1. Let $X$ be a compact subset of a Hausdorff topological space and 7: $X \rightarrow X$ a commutative semigroup of self-mappings. Let $x \in X$; then there is a point $z \in X$ with the property that for each $f \in \mathcal{Y}$, there is a net $\left\{g_{\alpha} f(x)\right\}$ (with $\alpha \in u_{z}$, the family of all neighbourhoods of $z$ directed by " $\subseteq$ ") converging to $z$, where $g_{\alpha} \in \mathcal{I}$.

Proof. For every $x \in X$, the collection $\{\exists f(x): f \in \exists\}$ has finite intersection property (by the commutativity of $\exists$ ). Since $X$ is a compact subset, $\bigcap_{f \in \mathcal{G}} \overline{\exists f(x)}$ $\neq \varnothing$. Let $z$ be a point in the intersection; then for each $f \in \exists, z \in \bar{\exists}(x)$, i.e., $z$ is a closure point of the set $\left\{g f(x): g \in \mathcal{g}\right.$. Hence there is a net $\left\{g_{\alpha} f(x)\right\}$ in $\exists f(x)$ converging to $z$ for each fixed $f \in \mathcal{\exists}$ (with $\alpha \in u_{z}$, the family of all neighbourhoods of $z$ directed by " $\subseteq$ "). This proves the lemma.

We denote the fact that the net $\left\{g_{\alpha} f(x)\right\}$ converges to $z$ by $\lim _{\alpha} g_{\alpha} f(x)=z$. It is clear that since $X$ is Hausdorff, $z$ is the unique point to which the net $\left\{g_{\alpha} f(x)\right\}$ in the lemma above converges. 
Lemma 2. Let $X$ be a convex subset of a linear topological space, and $\Im: X \rightarrow X$ be a commutative semigroup of asymptotically-affine, self-mappings. Then for every finite set of points $x_{1}, x_{2}, \ldots, x_{n} \in X$ and $\lambda_{i} \in[0,1], i=1, \ldots, n$ with $\sum_{i=1}^{n} \lambda_{i}$ $=1$, there is an $h \in \mathcal{Y}$ such that $f h\left(\sum_{i=1}^{n} \lambda_{i} x_{i}\right)=\sum_{i=1}^{n} \lambda_{i} f h\left(x_{i}\right)$.

Proof. For $n=2$, the conclusion is obvious by the hypothesis on 7 . Assume that the proposition is true for $n=k-1$, where $k \geq 3$. Let $x_{1}, \ldots, x_{k} \in X$ and $\lambda_{i} \in[0,1], i=1, \ldots, k$, with $\sum_{i=1}^{k} \lambda_{i}=1$. Since

$$
\sum_{i=1}^{k} \lambda_{i} x_{i}=\left(1-\lambda_{k}\right) \sum_{i=1}^{k-1}\left(\frac{\lambda_{i}}{1-\lambda_{k}}\right) x_{i}+\lambda_{k} x_{k}
$$

and $\exists$ is asymptotically-affine on $X$, there exists $g \in \exists$ such that the elements $\sum_{i=1}^{k-1}\left(\lambda_{i} /\left(1-\lambda_{k}\right)\right) x_{i}$ and $x_{k}$ are asymptotically-affine with respect to it. By the induction hypothesis, there exists $g^{\prime} \in \mathcal{Y}$ such that

$$
f g^{\prime} \sum_{i=1}^{k-1}\left(\frac{\lambda_{i}}{1-\lambda_{k}}\right) x_{i}=\sum_{i=1}^{k-1}\left(\frac{\lambda_{i}}{1-\lambda_{k}}\right) f g^{\prime}\left(x_{i}\right) \text { for all } f \in \mathcal{G}
$$

Hence $h=g g^{\prime}$ is a member in $\rightarrow$ such that

$$
f h \sum_{i=1}^{k} \lambda_{i} x_{i}=\sum_{i=1}^{k} \lambda_{i} f h\left(x_{i}\right) \text { for all } f \in \mathcal{Y},
$$

proving the assertion of the lemma.

1.2. Theorem 2. Let $X$ be a compact convex subset of a Hausdorff linear topological space, Э: $X \rightarrow X$ a commutative semigroup of continuous, asymptotically-affine self-mappings. Then $\Im$ has a common fixed point.

Proof. Let $x \in X$. By Lemma 1, there exists $z \in X$ with the property that for every $f \in \mathcal{Y}$, there is a net $\left\{g_{\alpha} f(x)\right\}$ (where $g_{\alpha} \in \mathcal{Y}$ ) converging to $z$, i.e. $\lim _{\alpha} g_{\alpha} f(x)=z$.

We will show that $\exists$ is affine on $\overline{c o} \Im(z)$. To this end it suffices to show that $\exists$ is affine on $\operatorname{co} \Im(z)$ :

Let $y=\sum_{i=1}^{n} \lambda_{i} g_{i}(z)$ where $g_{i} \in \mathcal{Y}$, for $i=1, \ldots, n$ and $\sum_{i=1}^{n} \lambda_{i}=1$. Let $h \in \exists$ be obtained by Lemma 2 with the property that $f h\left[\sum_{i=1}^{n} \lambda_{i} g_{i}(x)\right]$ $=\sum_{i=1}^{n} \lambda_{i} f h g_{i}(x)$ for every $f \in \mathcal{G}$. Since $z=\lim _{\alpha} g_{\alpha} h(x)$, we have

$$
\begin{aligned}
f(y) & =f\left[\sum_{i=1}^{n} \lambda_{i} g_{i}(z)\right]=f\left[\sum_{i=1}^{n} \lambda_{i} g_{i}\left[\lim _{\alpha} g_{\alpha} h(x)\right]\right] \\
& =\lim _{\alpha} f g_{\alpha} h\left[\sum_{i=1}^{n} \lambda_{i} g_{i}(x)\right]=\sum_{i=1}^{n} \lambda_{i} f\left[\lim _{\alpha} g_{\alpha} h g_{i}(x)\right] \\
& =\sum_{i=1}^{n} \lambda_{i} f g_{i}(z) \quad \text { for every } f \in \mathcal{Y} .
\end{aligned}
$$


Let $\sum_{i=1}^{n} \lambda_{i} g_{i}(z)$ (where $\left.\sum_{i=1}^{n} \lambda_{i}=1\right)$ and $\sum_{i=1}^{n^{\prime}} \gamma_{i} g_{i}(z)$ (where $\sum_{i=1}^{n^{\prime}} \gamma_{i}=1$ ) be two elements in co $\Im(z)$. For any $\beta \in[0,1]$, since $\beta\left(\sum_{i=1}^{n} \lambda_{i} g_{i}(z)\right)+(1-\beta)$ - $\left(\sum_{i=1}^{n^{\prime}} \gamma_{i} g_{i}(z)\right)$ is an element in co $\Im(z)$, we have

$$
\begin{aligned}
f\left[\beta\left(\sum_{i=1}^{n} \lambda_{i} g_{i}(z)\right)\right. & \left.+(1-\beta)\left(\sum_{i=1}^{n^{\prime}} \gamma_{i} g_{i}(z)\right)\right] \\
= & \beta\left(\sum_{i=1}^{n} \lambda_{i} f g_{i}(x)\right)+(1-\beta) \sum_{i=1}^{n^{\prime}} \gamma_{i} f g_{i}(z) \\
= & \beta\left(f \sum_{i=1}^{n} \lambda_{i} g_{i}(z)\right)+(1-\beta)\left(f \sum_{i=1}^{n^{\prime}} \gamma_{i} g_{i}(z)\right) .
\end{aligned}
$$

Hence, $\Im$ is a commutative semigroup of affine mappings of co $\Im(z)$ into itself. Now $\overline{c o} \Im(z)$ is clearly a compact convex subset of $X$. By the Markov-Kakutani theorem, there is a common fixed point in $\overline{c o} \ni(z)$.

2. Fixed point theorems for commutative semigroups of continuous asymptotically-nonexpansive self-mappings are obtained in this section.

$\exists$ is called a family of asymptotically-nonexpansive mappings (a family of asymptotic isometries) or simply asymptotically-nonexpansive (asymptotically-isometric) if for every $x, y \in X$, there exists $g \in \exists$ such that

$$
\begin{aligned}
d[f g(x), f g(y)] & \leq d(x, y), \\
(d[f g(x), f g(y)] & =d(x, y)) \quad \text { for every } f \in \mathcal{\exists} .
\end{aligned}
$$

(Examples of commutative asymptotically-nonexpansive semigroups which fail to be nonexpansive are given in \$4.)

The set $\{z \in X$ : there exists $x \in X$ such that for every $f \in \mathcal{F}, \varepsilon>0$, there exists $g \in \mathcal{Y}$ with $d[f g(x), z]<\varepsilon\}$ is called the $\mathcal{Y}$-closure of $X$ and is denoted by $X^{?}$.

2.1. We state first a result by Holmes and Narayanaswami [6] concerning asymptotically-nonexpansive semigroups of self-mappings which will be used subsequently.

Theorem 3 (Holmes and Narayanaswami [6]). Let $(X, d)$ be a metric space, and $\exists$ a commutative semigroup of continuous asymptotically-nonexpansive mappings on $X$.

(i) If $z \in X^{\Im}$, then $\Im \mid \neg(z)$ is a family of isometries.

(ii) If $A$ is an arbitrary subset of $X$ with the property that $\exists \mid A$ is a family of asymptotic isometries, then $\Im \mid A$ is a family of isometries.

(iii) If $z \in X^{\mathcal{9}}$, then for every $f \in \mathcal{\Im}, \varepsilon>0$, there exists $g \in \mathcal{\Im}$ such that $d[f g(z), z]<\varepsilon$.

The following result by Holmes, which is a generalization of a theorem by De Marr, will be compared with a corollary of the main result in this section: 
Theorem 4 (Holmes [4]). Let $X$ be a nonempty compact convex subset of a Banach space. If $\Im: X \rightarrow X$ is a commutative semigroup of continuous asymptoticallynonexpansive mappings, then $\Im$ has a fixed point in $X$.

2.2. A convex subset $C$ in a Banach space $X$ is said to have normal structure if for each bounded convex subset $K$ of $C$ which contains more than one point there exists a point $x$ in $K$ which is not a diametral point of $K$. (A point $x$ in $X$ is said to be a diametral point of $K$ if $\sup \{\|x-y\|: y \in K\}=d$ where $d$ is the diameter of $K$.)

Let $C$ be a closed convex and bounded subset of a Banach space $X$. Assume furthermore that $C$ is weakly compact and has normal structure. (It was shown in [2] that the latter assumption is automatically satisfied when $C$ is compact and convex.)

The concept of the centre of $C$ was introduced by Brodskir and Milman in [1]. It was shown there that the centre of a closed convex, bounded and weakly compact subset $C$ with normal structure of a Banach space $X$ is a common fixed point of all isometric mappings of $C$ onto itself.

2.3. Let $X$ be a compact subset of a general metric space. Suppose $\Im$ is a commutative semigroup of self-mappings on $X$. Since for each $x \in X$, the collection $\{\overline{\exists f(x)}: f \in \mathcal{Y}\}$ of closed subsets in $X$ has the finite intersection property, $\bigcap_{f \in \mathcal{S}} \overline{\exists f(x)} \neq \varnothing$. Hence $X^{\Im} \neq \varnothing$. On the other hand, there are examples of noncompact metric spaces $X$ with commutative semigroups $\rightarrow$ of self-mappings on it where $X^{\Im} \neq \varnothing$ (see Example 4.2 in $\left.\$ 4\right)$. This idea motivates the investigation of fixed point theorems for semigroups $\Im$ of asymptoticallynonexpansive self-mappings on a subset $X$ of a Banach space where $X^{\mathfrak{9}} \neq \varnothing$.

The following result is obtained.

Theorem 5. Let $X$ be a weakly compact, convex subset of a strictly convex Banach space, Э: $X \rightarrow X$ a commutative semigroup of continuous asymptotically-nonexpansive mappings. Suppose $X^{\Im} \neq \varnothing$. Let $z \in X^{\Im}$; then $\Im$ has a common fixed point in $\overline{\mathrm{co}} \ni(z)$.

2.4. The approach used in the proof of Theorem 5 is a generalization of that used by Edelstein in a theorem in [3]. We proceed first by defining a mapping from $\exists \times \mathbf{N}$ to $\exists$. This is followed by a few lemmas which are used in the proof of Theorem 5.

By the definition of $X^{9}$ and Theorem 3(iii), if $z \in X^{9}$, then $z$ has the property that for every $n \in N, f \in \mathcal{Y}$, there exists $g \in \mathcal{Y}$ such that $\|z-g f(z)\|<1 / n$. Now, for each pair $(f, n) \in \Im \times N$, we make a fixed choice of $g \in \mathcal{Y}$ which satisfies the above inequality. We obtain then a mapping $\gamma: \mathfrak{\exists} \times \mathbf{N} \rightarrow \mathfrak{I}$ with $\gamma(f, n)=\gamma_{f, n}$ satisfying $\left\|z-\gamma_{f, n} f(z)\right\|<1 / n$.

Let $S=\left\{x \in X\right.$ : for every $\left.(f, n),\left\|x-\gamma_{f, n} f(x)\right\|<1 / n\right\}$. Since $z \in S, S \neq \varnothing$.

2.5. For this set $S$ which is obtained when $\Im$ is a commutative semigroup of continuous, asymptotically-nonexpansive mappings we prove the following lemmas: 
Lemma 1. For every $g \in \mathcal{G}, g(S) \subseteq S$.

Proof. For every $x \in S$, by Theorem 3(i), $\Im \mid \mathcal{Y}(x)$ is a family of isometries. By the definition of $S,\left\|x-\gamma_{f, n} f(x)\right\|<1 / n$ for all $(f, n) \in \exists \times N$. Hence we have $\left\|g(x)-\gamma_{f, n} f g(x)\right\|<1 / n$, showing that $g(x) \in S$.

Lemma 2. $\mathcal{\exists} \mid \bar{S}$ is a family of isometries.

Proof. Since $\Im$ is commutative, by Theorem 3(ii), it suffices to show that $\exists \mid S$ is a family of asymptotic isometries.

Suppose the contrary; then there exist $x_{1}, x_{2} \in S$ with the property that for every $f \in \mathcal{Y}$, there exists $g_{f} \in \mathcal{Y}$ such that $\left\|f g_{f}\left(x_{1}\right)-f g_{f}\left(x_{2}\right)\right\| \neq\left\|x_{1}-x_{2}\right\|$.

Since $\Im$ is asymptotically-nonexpansive, there is an $h \in \mathcal{G}$ such that $\| f h\left(x_{1}\right)$ $-f h\left(x_{2}\right)\|\leq\| x_{1}-x_{2} \|$ for every $f \in \mathcal{F}$. For this $h$, let $g$ be a member in $\mathcal{\exists}$ such that $\left\|h g\left(x_{1}\right)-h g\left(x_{2}\right)\right\| \neq\left\|x_{1}-x_{2}\right\|$ is satisfied. Then

$$
\left\|h g\left(x_{1}\right)-h g\left(x_{2}\right)\right\|<\left\|x_{1}-x_{2}\right\| .
$$

For the pair of points $h g\left(x_{1}\right)$ and $h g\left(x_{2}\right)$, there exists $t \in \mathcal{F}$, such that for every $f \in \mathcal{Y}$,

$$
\left\|f \operatorname{th} g\left(x_{1}\right)-f \operatorname{th} g\left(x_{2}\right)\right\| \leq\left\|h g\left(x_{1}\right)-h g\left(x_{2}\right)\right\|<\left\|x_{1}-x_{2}\right\| .
$$

If we denote $g_{0}=t h g,(2)$ becomes

$$
\left\|f g_{0}\left(x_{1}\right)-f g_{0}\left(x_{2}\right)\right\| \leq\left\|h g\left(x_{1}\right)-h g\left(x_{2}\right)\right\|<\left\|x_{1}-x_{2}\right\|
$$

for every $f \in \mathcal{Y}$. Now for each $n$, we have

$$
\begin{aligned}
\left\|x_{1}-x_{2}\right\| \leq & \left\|x_{1}-\gamma_{g_{0}, n} g_{0}\left(x_{1}\right)\right\| \\
& +\left\|\gamma_{g_{0}, n} g_{0}\left(x_{1}\right)-\gamma_{8_{0}, n} g_{0}\left(x_{2}\right)\right\|+\left\|x_{2}-\gamma_{8_{0}, n} g_{0}\left(x_{2}\right)\right\| \\
< & 2 / n+\left\|h g\left(x_{1}\right)-h g\left(x_{2}\right)\right\| .
\end{aligned}
$$

Since $n$ is arbitrary, (3) and (1) together imply that $\left\|x_{1}-x_{2}\right\| \leq \| h g\left(x_{1}\right)$ $-h g\left(x_{2}\right)\|<\| x_{1}-x_{2} \|$, which is a contradiction.

Hence $\exists \mid S$ is a family of isometries. Consequently $\exists \mid \bar{S}$ is also a family of isometries.

Lemma 3. If $X$ is a convex subset of a strictly convex Banach space, then for every $f \in \mathcal{G}, f\left[\lambda x_{1}+(1-\lambda) x_{2}\right]=\lambda f\left(x_{1}\right)+(1-\lambda) f\left(x_{2}\right)$ holds for every $x_{1}, x_{2} \in \bar{S}$ and $\lambda \in[0,1]$.

Proof. Let $x_{1}, x_{2} \in S$ and suppose $x=\lambda x_{1}+(1-\lambda) x_{2}$ where $\lambda \in[0,1]$. We first show that there exists a $g \in \mathcal{\exists}$ (depending on $x_{1}, x_{2}, \lambda$ ) such that $f g x$ $=\lambda f g\left(x_{1}\right)+(1-\lambda) f g\left(x_{2}\right)$ for every $f \in \mathcal{G}$ : 
Since $\exists$ is commutative and asymptotically-nonexpansive, there exists $g \in \mathcal{\exists}$ depending on $x_{i}(i=1,2)$ and $x$ (i.e. depending on $x_{1}, x_{2}$ and $\lambda$ ) such that $\left\|f g\left(x_{i}\right)-f g(x)\right\| \leq\left\|x_{i}-x\right\|,(i=1,2)$, for all $f \in \mathcal{\exists}$.

Now

$$
\begin{aligned}
\left\|x_{1}-x\right\| & =\left\|x_{1}-x_{2}\right\|-\left\|x-x_{2}\right\| \\
& \leq\left\|f g\left(x_{1}\right)-f g\left(x_{2}\right)\right\|-\left\|f g(x)-f g\left(x_{2}\right)\right\| \\
& \leq\left\|f g\left(x_{1}\right)-f g(x)\right\| \leq\left\|x_{1}-x\right\|
\end{aligned}
$$

for every $f \in \mathcal{Y}$ (by applying Lemma 2).

The above inequalities imply that for every $f \in \mathcal{Y}$ we have

$$
\left\|x_{1}-x\right\|=\left\|f g\left(x_{1}\right)-f g(x)\right\|
$$

and

$$
\left\|f g\left(x_{1}\right)-f g\left(x_{2}\right)\right\|=\left\|f g(x)-f g\left(x_{2}\right)\right\|+\left\|f g\left(x_{1}\right)-f g(x)\right\| .
$$

By a similar argument as above, we obtain also

$$
\left\|x_{2}-x\right\|=\left\|f g\left(x_{2}\right)-f g(x)\right\| \text { for every } f \in \mathcal{Y} \text {. }
$$

By strict convexity (2) implies that

$$
f g(x)-f g\left(x_{2}\right)=\frac{\left\|f g x-f g x_{2}\right\|}{\left\|f g x_{1}-f g x\right\|}\left(f g\left(x_{1}\right)-f g(x)\right) .
$$

Hence,

$$
f g(x)-f g\left(x_{2}\right)=\frac{\left\|x_{2}-x\right\|}{\left\|x_{1}-x\right\|}\left(f g\left(x_{1}\right)-f g(x)\right) \quad \text { by (1) and (1') }
$$

Consequently, $f g(x)-f g\left(x_{2}\right)=(\lambda /(1-\lambda))\left(f g\left(x_{1}\right)-f g(x)\right)$ which implies that $f g(x)=\lambda f g\left(x_{1}\right)+(1-\lambda) f g\left(x_{2}\right)$ for every $f \in \mathcal{G}$.

Next, we observe that for every $x_{1}, x_{2} \in S$, and $\lambda \in[0,1]$, if $h \in \mathcal{\exists}$ (depending on $x_{1}, x_{2}, \lambda$ ) is chosen to satisfy

$$
f h\left[\lambda x_{1}+(1-\lambda) x_{2}\right]=\lambda f h\left(x_{1}\right)+(1-\lambda) f h\left(x_{2}\right) \quad \text { for every } f \in \mathcal{\Im},
$$

then

$$
\begin{aligned}
f\left[\lambda x_{1}+(1-\lambda) x_{2}\right] & =f\left[\lambda \lim _{n \rightarrow \infty} \gamma_{h, n} h\left(x_{1}\right)+(1-\lambda) \lim \gamma_{h, n} h\left(x_{2}\right)\right] \\
& =\lim _{n \rightarrow \infty}\left\{f \gamma_{h, n} h\left[\lambda x_{1}+(1-\lambda) x_{2}\right]\right\} \\
& =\lim \left\{\lambda f \gamma_{h, n} h\left(x_{1}\right)+(1-\lambda) f \gamma_{h, n} h\left(x_{2}\right)\right\} \\
& =\lambda f\left(x_{1}\right)+(1-\lambda) f\left(x_{2}\right)
\end{aligned}
$$


by the continuity of mappings in 7 .

It is immediate that the above equality holds when $x_{1}, x_{2} \in \bar{S}$. Hence the assertion of the lemma follows.

Lemma 4. Suppose $X$ is a convex subset of strictly convex Banach space; then $\bar{S}$ is convex.

Proof. It suffices to show that $S$ is convex. Let $x_{1}, x_{2} \in S$. For any choice $(f, n)$, we have

$$
\left\|x_{1}-\gamma_{f, n} f\left(x_{1}\right)\right\|<1 / n \text { and }\left\|x_{2}-\gamma_{f, n} f\left(x_{2}\right)\right\|<1 / n .
$$

Let $x=\lambda x_{1}+(1-\lambda) x_{2}$ where $\lambda \in[0,1]$. By Lemma 3, we have $f(x)=\lambda f\left(x_{1}\right)$ $+(1-\lambda) f\left(x_{2}\right)$ for every $f \in \mathcal{G}$, and $\lambda \in[0,1]$. Hence

$$
\begin{aligned}
\left\|x-\gamma_{f, n} f(x)\right\| & =\left\|\lambda x_{1}+(1-\lambda) x_{2}-\left[\lambda \gamma_{f, n} f\left(x_{1}\right)+(1-\lambda) \gamma_{f, n} f(x)\right]\right\| \\
& \leq \lambda\left\|x_{1}-\gamma_{f, n} f\left(x_{1}\right)\right\|+(1-\lambda)\left\|x_{2}-\gamma_{f, n} f\left(x_{2}\right)\right\|<1 / n .
\end{aligned}
$$

This shows that $x \in S$. Consequently $S$ and therefore $\bar{S}$ is convex.

2.6. Proof of Theorem 5. For the point $z \in X^{9}$, define a subset $S$ as in the discussion above. By Lemmas $1-4, \mathcal{Y} \mid \bar{S}$ is a commutative semigroup of affine isometries from the closed convex subset $\bar{S}$ into itself. Since $X$ is weakly compact, so is $\bar{S}$. Now $\mathcal{\exists} \mid \bar{S}$ is a family of affine isometries on a convex subset; hence each member in $\mathcal{Y} \mid \bar{S}$ is weakly continuous.

Since $z \in S$, the set $\mathcal{Y}(z)$ is in $S$ (by Lemma 1 ). As $\bar{S}$ is closed and convex, we have $\overline{c o} \Im(z) \subseteq \bar{S}$. Hence $\Im \mid \overline{c o} \Im(z)$ is a commutative semigroup of affine isometries on $\overline{c o} \Im(z)$. By Lemma 3, each $f \in \mathcal{Y}$ maps $\overline{c o} \Im(z)$ into itself. Since $\overline{\text { co }} \Im(z)$ is a weakly compact convex subset, we obtain a common fixed point of $\Im$ in $\overline{c o} \Im(z)$ by the Markov-Kakutani theorem.

\subsection{Corollaries to Theorem 5 .}

Corollary 1. Let $X, \Im, z$, be as in Theorem 5. Let in addition $X$ have normal structure and suppose $c$ is the centre of $\overline{c o} \ni(z)$. Then $c$ is a common fixed point of $\exists$.

Proof. Since $z \in X^{\mathfrak{9}}$, for every $f \in \mathcal{Y}$, there is a sequence $\left\{g_{n}\right\} \in \mathcal{Y}$ such that $z=\lim _{n \rightarrow \infty} g_{n} f(z)$. Since $\ni \mid \mathcal{Y}(z)$ is a family of isometries, $\left\|g_{m}(z)-g_{n}(z)\right\|$ $=\left\|g_{m} f(z)-g_{n} f(z)\right\|$, which shows that the sequence $\left\{g_{n}(z)\right\}$ is Cauchy. Consequently,

$$
z=\lim _{n \rightarrow \infty} g_{n} f(z)=f \lim _{n \rightarrow \infty} g_{n}(z)=f(t) \text { for some } t \in \overline{\Im(z)} .
$$

We proceed first by showing that $\exists: \overline{\exists(z)} \rightarrow \overline{\exists(z)}$ is a family of onto mappings. Let $x \in \overline{\mathcal{Y}(z)}$; then $x=\lim _{n \rightarrow \infty} h_{n}(z)$ for some sequence $\left\{h_{n}\right\} \in \mathcal{Y}$. By the fact that $t \in \overline{\mathcal{Y}(z)} \subseteq \bar{S} \subseteq X^{9}$ (where the subset $S$ is as in the proof of Theorem 3). and using a similar argument as above, $\lim _{n \rightarrow \infty} h_{n}(t)$ exists. Hence

$$
x=\lim _{n \rightarrow \infty} h_{n}(z)=\lim _{n \rightarrow \infty} h_{n} f(t)=f \lim _{n \rightarrow \infty} h_{n}(t) \in f \overline{\mathcal{Y ( z )}} .
$$


This shows that every $f$ in $\exists$ maps $\overline{\Im(z)}$ onto itself. Hence, for every $x \in \overline{c o} \Im(z)$

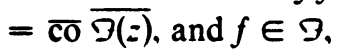

$$
x=\lim _{n \rightarrow \infty}\left\{\sum_{i=1}^{K_{n}} \lambda_{n, i} f\left(z_{n, i}\right)\right\}, \quad \text { where } z_{n, i} \in \overline{\Im(z)} \text {, and } \sum_{i=1}^{K_{n}} \lambda_{n, i}=1 .
$$

Since $f$ is affine on $\overline{\operatorname{co}} \Im(z)$, for each $n$,

$$
\sum_{i=1}^{K_{n}} \lambda_{n, i} f\left(z_{n, i}\right)=f\left[\sum_{i=1}^{K_{n}} \lambda_{n, i}\left(z_{n, i}\right)\right]
$$

Now the existence of $\lim _{n \rightarrow \infty}\left\{\sum_{i=1}^{K_{n}} \lambda_{n, i} f\left(z_{n, i}\right)\right\}$ implies that $\lim _{n \rightarrow \infty}\left\{\sum_{i=1}^{K_{n}} \lambda_{n, i}\left(z_{n, i}\right)\right\}$ exists, since $f$ is an affine isometry on $\overline{c o} \ni(z)$.

By the continuity of $f$, we obtain

$$
\begin{aligned}
x & =\lim _{n \rightarrow \infty}\left\{f\left[\sum_{i=1}^{K_{n}} \lambda_{n, i}\left(z_{n, i}\right)\right]\right\} \\
& =f\left[\lim _{n \rightarrow \infty}\left\{\sum_{i=1}^{K_{n}} \lambda_{n, i}\left(z_{n, i}\right)\right\}\right] \in f[\overline{\operatorname{co}} \ni(z)] .
\end{aligned}
$$

Consequently each $f \in \Im$ maps $\overline{c o} \Im(z)$ onto itself. As $\overline{c o} \Im(z)$ is a weakly compact convex subset of $x$ and $\exists$ is a family of weakly continuous, affine isometries from $\overline{c o} \Im(z)$ onto itself, by a result of Brodskii and Milman [1], the centre of $\overline{\operatorname{co}} \Im(z)$ is a common fixed point of $\exists$.

Corollary 2 (cf. Theorem 4). Let $X$ be a compact, convex subset of a strictly convex Banach space, Э: $X \rightarrow X$ be a commutative semigroup of asymptoticallynonexpansive mappings. Then for each $z \in X^{9}$, the centre of $\overline{c 0} \Im(z)$ is a common fixed point of 3 .

Proof. This follows immediately from Corollary 1 since each compact convex subset of a Banach space is weakly compact and has normal structure.

2.8. The following example shows that when strict convexity is deleted from the hypotheses of Corollary 2 to Theorem 5 , the assertion that the centre of $\overline{c o} \ni(z)$ (where $z \in X^{9}$ ) is a common fixed point of $\exists$ no longer holds.

Example. Let $X$ be the closed unit ball of $E^{2}$ with sup norm. Then $X$ is clearly compact and convex.

For every $p=(a, b)$, let $f: X \rightarrow X$ be defined by $f(p)=f(a, b)=(|b|,-b)$. Then $f$ is nonexpansive. Indeed,

$$
\begin{aligned}
\|f(a, b)-f(c, d)\| & =\|(|b|,-b)-(|d|,-d)\| \\
& =\max \{|b|-|d|,|b-d|\}=|b-d| \\
& \leq \max \{|a-c|,|b-d|\}=\|(a, b)-(c, d)\| .
\end{aligned}
$$


Let $\exists$ be the semigroup generated by $f$. The point $p=(1,1)$ is clearly in the $\exists$-closure of $X$. The orbit of $p$ under $\exists$ consists of only two points, $(1,1)$ and $(1,-1)$. However, the closed convex hull of these two points does not contain any fixed point of $f$. This shows that strict convexity is essential in the hypotheses of Corollary 2 to Theorem 5 .

3. Let $\Im$ be a commutative semigroup of nonexpansive or continuous asymptotically-nonexpansive mappings on a compact convex subset $X$ of a Banach space. The existence of a common fixed point has been established by De Marr [2] and Holmes [4] respectively. Corollary 2 to Theorem 5 shows that if the Banach space in question is furthermore strictly convex, then for every $z \in X^{\mathfrak{9}}$, the centre of $\overline{c o} \Im(z)$ is such a common fixed point. While the example following Corollary 2 shows that when strict convexity is deleted, the set co $\Im(z)$ (where $z \in X^{9}$ ) need not contain any common fixed point, it suggests a certain relation between $\mathcal{Y}(z)$ and such a point. We show that "the set of centres" in $X$ with respect to $\overline{Y(z)}$ does contain a common fixed point. This result also provides alternate proofs to the theorems obtained by De Marr and Holmes. Furthermore, it is shown that when $\operatorname{dim} X \leq n$, this alternate approach enables us to construct such a fixed point by at most $n$ successive steps.

3.1. Let $X$ be any bounded subset of a Banach space. Suppose $A \subseteq X$, for any $x \in X$; let

$$
\begin{aligned}
r_{A}(x) & =\sup \{\|x-y\|: y \in A\} \\
R & =\inf \left\{r_{A}(x): x \in X\right\}
\end{aligned}
$$

and

$$
C_{A}=\left\{u \in X: r_{A}(u)=R\right\}
$$

The set $C_{A}$ is called the set of centres in $X$ with respect to $A$.

When $A=X$ and $X$ is a convex subset of a uniformly convex Banach space, then the set of centres in $X$ with respect to $A$ is a singleton which coincides with the centre of a subset introduced by Brodskii and Milman. Since a uniformly convex Banach space has normal structure, the centre of such a set is a fixed point for any self-mapping which is an onto isometry.

In general, $C_{A}$ could be empty. However, when $X$ is compact and convex, $C_{A}$ is necessarily nonempty and closed. Suppose $\Im: X \rightarrow X$ is a commutative semigroup of continuous, asymptotically-nonexpansive mappings. Since $X^{9}$ $\neq \varnothing$, we can consider the restriction of $\Im$ on the compact subset $\overline{\mathcal{Y}(z)}$, where $z \in X^{\Im}$. By Theorem $3, \Im$ is a family of isometries of $\overline{\Im(z)}$ onto itself. It is from the set of centres with respect to $\overline{\mathcal{Y}(z)}$ that a common fixed point of $\Im$ will be constructed. 
3.2. Let Э: $X \rightarrow X$ and $Y \subseteq X$. The set $Y$ is called Э-invariant if $f(Y) \subseteq Y$ for every $f \in \Im$. A closed subset $Y \subseteq X$ is called $\Im$-stable if there is a collection $\mathcal{A}$ of closed subsets of $X$ such that (i) $Y=\cap \mathcal{A}$, (ii) for every $x \in Y, B \in \mathcal{A}$, there exists $g \in \mathcal{\exists}$ such that $f g(x) \in B$ for every $f \in \mathcal{\exists}$.

The following lemma is shown in [4] concerning 9 -stable subsets.

Lemma 1 (see [4]). (i) Any closed 9-invariant subset A is 9-stable.

(ii) An arbitrary intersection of 7-stable subsets is 7 -stable.

(iii) Suppose $A$ is $\rightarrow$-stable and $A$ is the corresponding collection of closed subsets. If $A$ contains a compact member, then there is a $z \in A$ such that the orbit $\Im(z)$ of $z$ is contained in $A$.

Lemma 2. Let $X$ be a compact convex subset of a Banach space, Э: $X \rightarrow X$ be a commutative semigroup of nonexpansive mappings. Suppose $z \in X^{9}$; then $C_{A}$ (where $A=\overline{\mathcal{Y}(z)})$ is a compact, convex $\mathcal{Y}$-invariant subset of $X$.

Proof. (i) Since $X$ is compact convex, $C_{A}$ is a nonempty closed, and therefore compact subset of $X$.

(ii) Let $c_{1}, c_{2} \in C_{A}$. By the definition of $C_{A}$, we have $r_{A}\left(c_{1}\right)=r_{A}\left(c_{2}\right)=R$ $=\inf \left\{r_{A}(x): x \in X\right\}$, i.e. $\sup \left\{\left\|c_{1}-y\right\|: y \in A\right\}=\sup \left\{\left\|c_{2}-y\right\|: y \in A\right\}=R$. Let $x=\lambda c_{1}+(1-\lambda) c_{2}$ where $\lambda \in[0,1]$. Since, for every $y \in A$,

$$
\begin{aligned}
\|x-y\| & =\left\|\left(\lambda c_{1}+(1-\lambda) c_{2}\right)-y\right\| \\
& \leq \lambda\left\|c_{1}-y\right\|+(1-\lambda)\left\|c_{2}-y\right\| \leq R,
\end{aligned}
$$

we have $\sup \{\|x-y\|: y \in A\}=R$. Hence $x \in C_{A}$ showing that $C_{A}$ is convex.

(iii) By Theorem 3, and since $A$ is compact, $\Im \mid A$ is a family of onto isometries. Hence, for every $y \in A$ and $f \in \mathcal{\Im}$, we have $y=f(a)$ for $a \in A$. Let $x \in C_{A}$, then $\|f(x)-y\|=\|f(x)-f(a)\| \leq\|x-a\| \leq R$ showing that $r_{A}(f(x)) \leq R$. By the definition of $R$, we have $R=r_{A}(f(x))$ which implies that $f(x) \in C_{A}$.

As this result holds for all $f \in \mathcal{\exists}$, the subset $C_{A}$ is $\mathcal{Y}$-invariant.

Lemma 3. Let $X$ be a compact convex subset of a Banach space, $\Im: X \rightarrow X$ be a commutative semigroup of continuous, asymptotically-nonexpansive mappings. Let $z \in X^{\Im}$; then $C_{A}($ where $A=\overline{\mathcal{Y}(z)})$ is a compact convex subset which is 9 -stable.

Proof. Since $X$ is compact convex, $C_{A}$ is nonempty, closed, and therefore compact. It remains to show that $C_{A}$ is $\mathcal{Y}$-stable.

Now, $R=\inf \left\{r_{A}(x): x \in X\right\}$ implies that

$$
C_{A}=\bigcap_{\varepsilon>0}\left[\bigcap_{z \in A} \bar{B}(z, R+\varepsilon)\right] \cap X .
$$

For any fixed $x \in C_{A}$ and $\varepsilon>0$, let $\left\{W_{g}\right\}_{g \in \mathcal{O}}$ be a collection of subsets in $A$ where

$$
W_{g}=\{z \in A: \sup \{\|f g(x)-f g(z)\|: f \in \Im\}<\|x-z\|+\varepsilon\} .
$$


Using the fact that each $f \in \mathcal{Y}$ is an isometry of $A=\overline{\mathcal{Y}(z)}$ onto itself, it can be shown that $\left\{W_{8}\right\}_{g \in O}$ is an open covering of $A$ (see the proof of the main theorem in [4]). Since $A$ is compact, we obtain a finite subcover of $A$, say $W_{81}, \ldots, W_{g_{N}}$. The mapping $g=g_{1} \cdot \ldots \cdot g_{N} \in \mathcal{G}$ satisfies the condition that $f g(x) \in$ $\bar{B}(z, R+\varepsilon)$, for every $f \in \mathcal{Y}$ and $z \in A$. Indeed, for every $z \in A, z=f g_{1} \cdot \ldots$ $\cdot g_{N}\left(z^{\prime}\right)$ for some $z^{\prime} \in \overline{\mathcal{Y}(z)}$; therefore

$$
\left\|f g_{1} \cdot \ldots \cdot g_{N}(x)-z\right\|=\left\|f g_{1} \cdot \ldots \cdot g_{N}(x)-f g_{1} \cdot \ldots \cdot f_{N}\left(z^{\prime}\right)\right\| \leq R+\varepsilon .
$$

Lemma 4. Let $X, \exists, A, C_{A}$ be as in Lemma 2 (or Lemma 3 ); then $C_{A}$ has an empty interior.

Proof. Let $x \in C_{A}$. Suppose, for some $\varepsilon>0$, there is $B(x, \varepsilon)$ with $B(x, \varepsilon) \subseteq C_{A}$. Now $x \in C_{A}$ implies that $r_{A}(x)=R$ (where $R=\inf \left\{r_{A}(x): x \in X\right\}$ ). By the compactness of $A$, there is a point $y_{0} \in A$ such that $\left\|x-y_{0}\right\|=R$. Hence there exists $z \in B(x, \varepsilon)$ such that $\left\|z-y_{0}\right\|=R+\delta$ for some $\delta$ satisfying $0<\delta<\varepsilon$. This implies that $r_{A}(z) \geq R+\delta$, or $r_{A}(z) \notin C_{A}$, contradicting the assumption that $B(x, \varepsilon) \subseteq C_{A}$. Consequently, $C_{A}$ has an empty interior.

3.3. Theorem 6. Let $X$ be a compact convex subset of a Banach space, $9: X \rightarrow X$ be a commutative semigroup of nonexpansive mappings. Let $z \in X^{9}$; then there is a common fixed point of $\exists$ in $C_{A}$ (where $\left.A=\overline{\exists(z)}\right)$.

Proof. By Lemma 2, $C_{A}$ is a compact convex subset which is $\Im$-invariant. Since $X$ is compact convex, $R=\inf r_{A}(x)<\operatorname{diam} X$ (by a lemma in [2]). Hence $C_{A}$ is a proper subset of $X$.

Denote $X=X_{1}$ and $C_{A}=X_{2}$, apply Lemma 2 to $X_{2}$; we obtain a proper compact convex subset in $X_{2}$, denoted by $X_{3}$, which is again $\Im$-invariant.

Using induction, one obtains a transfinite sequence $X_{1}, X_{2}, \ldots, X_{\omega}, X_{\omega+1}, \ldots$ where each $X_{\beta}$ is properly contained in $X_{\alpha}$ whenever $\beta>\alpha$, for all ordinal numbers $\beta$ and $\alpha$. Consequently, $X_{\alpha}$ is a singleton for some ordinal number $\alpha$. Since $X_{\alpha}$ is also $\exists$-invariant, it is the required common fixed point.

Corollary 1. Let $X, \exists, z, C_{A}$ be as in Theorem 6. Suppose $\operatorname{dim} X \leq n$; then a common fixed point for $\exists$ can be constructed from $C_{A}$ by at most $n$ successive steps.

Proof. By Lemma 4, $C_{A}$ has an empty interior. Since $\operatorname{dim} X \leq n$, and as $C_{A}$ consists of just boundary points itself, $\operatorname{dim} C_{A} \leq n-1$. Now $C_{A}$ is a compact, convex, $\mathcal{F}$-invariant subset. By Lemma 2 we obtain a proper, compact, convex subset of $C_{A}$, say $X_{2}$, with $\operatorname{dim} X_{2} \leq n-2$. Hence, after at most $n$ steps, we obtain a $\mathcal{Y}$-invariant convex, compact subset $X_{n}$ with $\operatorname{dim} X_{n} \leq 0$. Thus $X_{n}$ is necessarily a singleton, which is a common fixed point of $\exists$.

Theorem 7. Let $X, C_{A}$ be as in Theorem 6. Let $\exists: X \rightarrow X$ be a commutative semigroup of continuous, asymptotically-nonexpansive mappings. Then there is a common fixed point of $\exists$ in $C_{A}$. 
Proof. By Lemma 3, $C_{A}$ is a compact, convex, 7 -stable subset of $X$. It is also a proper subset of $X$ (by the same argument used in the proof of Theorem 6).

Since $C_{A}$ is $\Im$-stable, there is a $z_{0} \in C_{A}$ such that $\overline{\mathcal{Y}\left(z_{0}\right)} \subseteq C_{A}$ by Lemma 1 .

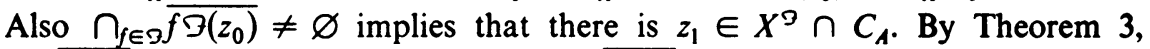
$\Im \mid \overline{\Im\left(z_{1}\right)}$ is a family of isometries of $\overline{\mathcal{Y}\left(z_{1}\right)}$ onto itself. Let $X_{1}=C_{A}$ and $A_{2}=\overline{\exists\left(z_{1}\right)}$. Suppose $C_{A_{2}}$ denotes the set of centres in the convex compact subset $X_{1}$ with respect to $A_{2}$. By applying the same argument as before, $C_{A_{2}}$ is a proper, compact, convex, 7 -stable subset of $X_{1}$. Using transfinite induction one obtains a $\exists$-stable subset which is a singleton. By Lemma 1(iii), this singleton is necessarily a common fixed point of $\exists$.

Corollary 2. Let $X, \Im, C_{A}$ be as in Theorem 7. Suppose $\operatorname{dim} X \leq n$; then a common fixed point of $\exists$ can be constructed by at most $n$ successive steps.

The proof is similar to the proof of Corollary 1 .

4. Examples.

4.1. Let $X$ be a normed linear space. Let $f$ be a mapping defined as follows:

$$
\begin{aligned}
f(x) & =\overline{0}, & & \text { for }\|x\| \leq 1, \\
& =\left(\frac{\|x\|-1}{\|x\|}\right) x, & & \text { for }\|x\|>1 ;
\end{aligned}
$$

i.e. $f$ is a mapping that maps the closed unit ball to the origin and moves vectors outside the unit ball radially towards the origin to $f(x)$ with $\|f(x)\|=\|x\|-1$.

It is clear that $f$ is not an affine mapping. The semigroup $\rightarrow$ generated by $f$ is, however, asymptotically-affine.

4.2. Let $X$ be a normed linear space. For $x \in X$, define $f: X \rightarrow X$ as follows:

$$
\begin{array}{ll}
f(x)=(1 / 4) x, & \text { for }\|x\| \leq 1, \\
f(x)=\left(\frac{(3 / 2)\|x\|-5 / 4}{\|x\|}\right) x, & \text { for } 1 \leq\|x\| \leq 3 / 2, \\
f(x)=\left(\frac{\|x\|-1 / 2}{\|x\|}\right) x, & \text { for }\|x\| \geq 3 / 2 .
\end{array}
$$

Hence $f$ is a mapping that "shrinks" the closed unit ball to the closed unit ball centred at the origin with radius $1 / 4$. For the annulus $\{x: 1 \leq\|x\| \leq 3 / 2\}$, the action of $f$ "expands" it to the annulus $\{x: 1 / 4 \leq\|x\| \leq 1\}$. For points outside the closed ball with radius $3 / 2$ centred at the origin, $f$ simply moves them radially towards the origin such that $\|f(x)\|=\|x\|-1 / 2$.

The mapping $f$ fails to be nonexpansive. However, for every pair of points $x, y \in X$, when $n \geq 2[\max \{\|x\|,\|y\|\}-1]$, both the points $x, y$ are inside the closed unit ball. Hence, after $f^{n}$, the action of $f$ on $f^{n}(x)$ and $f^{n}(y)$ becomes a 
t-contraction. Consequently, for $m$ sufficiently large, we have $\| f^{n} f^{m}(x)$ $-f^{n} f^{m}(y)\|\leq\| x-y \|$ for $n=1,2, \ldots$. This shows that the semigroup generated by $f$ is asymptotically-nonexpansive.

\section{REFERENCES}

1. M. S. Brodskii and D. P. Milman, On the center of a convex set, Dokl. Akad. Nauk SSSR $\$ 9$ (1948), 837-840. (Russian) MR 9, 448.

2. R. De Marr, Common fixed points for commuting contraction mappings, Pacific J. Math. 13 (1963), 1139-1141. MR 28 \#2446.

3. M. Edelstein, On nonexpansive mappings of Banach spaces, Proc. Cambridge Philos. Soc. 60 (1964), 439-447. MR 29 \# 1521.

4. R. D. Holmes, A generalization of a theorem of De Marr (unpublished).

5. R. D. Holmes and Anthony T. Lau, Asymptotically non-expansive actions of topological semigroups and fixed points, J. London Math. Soc. (2) 5 (1972), 330-336.

6. R. D. Holmes and P. P. Narayanaswami, On asymptotically nonexpansive semigroups of mappings, Canad. Math. Bull. 13 (1970), 209-214. MR 42 \# 1096.

7. S. Kakutani, Two fixed point theorems concerning bicompact convex subsets, Proc. Japan Acad. 14 (1938), 242-245.

8. A. Markov, Quelques théorèmes sur les ensembles abéliens, Dokl. Akad. Nauk SSSR 10 (1936), 311-314.

Department of Mathematics, St. Mary's University, Halifax, Nova Scotia, Canada 